\title{
Concentrating colloids with electric field gradients. II. Phase transitions and crystal buckling of long-ranged repulsive charged spheres in an electric bottle
}

\author{
Mirjam E. Leunissen ${ }^{\text {a) }}$ and Alfons van Blaaderen ${ }^{\text {b) }}$ \\ Soft Condensed Matter, Debye Institute for Nanomaterials Science, Utrecht University, Princetonplein 5 , \\ 3584 CC Utrecht, The Netherlands
}

(Received 10 December 2007; accepted 21 March 2008; published online 22 April 2008)

\begin{abstract}
We explored the usefulness of electric field gradients for the manipulation of the particle concentration in suspensions of charged colloids, which have long-ranged repulsive interactions. In particular, we studied the compression obtained by "negative" dielectrophoresis, which drives the particles to the regions of lowest field strength, thus preventing unwanted structural changes by induced dipole-dipole interactions. We used several sample cell layouts and suspension compositions, with a different range of the interparticle repulsions. For these systems, we obtained sufficient compression to observe a transition from the initial fluid phase to a random hexagonal close-packed crystal, as well as a body-centered cubic crystal. The heterogeneous dielectrophoretic crystallization mechanism involved an intriguing "pluglike" motion of the crystal, similar to what we have previously reported for hard-sphere suspensions. In this way, remarkably large single crystals were formed of several millimeters wide and a couple of centimeters long. Moreover, we found that these crystals could be compressed to such an extent that it led to an anisotropic deformation ("buckling") and, upon subsequent relaxation, a reorientation of the lattice, while stacking errors disappeared. These striking differences with the compressed hard-sphere crystals that we studied before [M. E. Leunissen et al., J. Chem. Phys. 128, 164508 (2008).] are likely due to the smaller elastic moduli of the present lower-density soft-sphere crystals. (C) 2008 American Institute of Physics. [DOI: 10.1063/1.2909200]
\end{abstract}

\section{INTRODUCTION}

An important factor that determines the phase behavior of all colloidal suspensions is the particle concentration or "volume fraction." For suspensions of the so-called hard spheres, which have no other interaction than a repulsive excluded volume interaction, ${ }^{1}$ it even is the only control parameter. For these suspensions, it has recently been demonstrated that dielectrophoretic forces ${ }^{2}$ generated by an electric field gradient form a powerful tool to control the particle concentration inside a sealed sample cell (an "electric bottle"). ${ }^{3,4}$ It was, for instance, shown that one can induce not only the formation of large crystals, with a random hexagonal close-packed ("rhcp") structure but also the melting, in a controlled way.

Here, we continue the exploration of the electric bottle concept, where one lets a confined colloidal suspension come to equilibrium under the action of an electric field gradient. In particular, we will focus on the manipulation of charged ("soft") spheres, which have long-ranged repulsive interactions. As a model system, we used suspensions of sterically stabilized polymethyl methacrylate (PMMA) particles (dielectric constant $\left.\varepsilon_{p} \approx 2.6\right)$ in a near density- and indexmatched mixture of the organic solvents cyclohexyl bromide

\footnotetext{
${ }^{a)}$ Present address: Center for Soft Matter Research, New York University, 4 Washington Place, New York, NY 10003, USA. Electronic mail: m.e.leunissen@nyu.edu.

b)Electronic mail: a.vanblaaderen@uu.nl.
}

and cis-decalin $\left(\varepsilon_{m} \approx 6-7\right)$. Based on our earlier investigations, ${ }^{5-7}$ we assume that these particles interact via a hard-core repulsive screened Coulomb (or "Yukawa") potential. For a pair of particles separated by a distance $r$, this interaction is given by

$$
\frac{u(r)}{k_{B} T}=\left\{\begin{array}{cl}
\gamma_{c} \frac{\exp [-\kappa \sigma(r / \sigma-1)]}{r / \sigma}, & r \geqslant \sigma \\
\infty, & r<\sigma
\end{array}\right.
$$

with $\gamma_{c}$ as the value of the potential at contact (per $k_{B} T$, where $k_{B}$ is Boltzmann's constant and $T$ is the absolute temperature), $\kappa$ as the inverse Debye screening length $\left(\kappa^{-1}\right.$ $=1 / \sqrt{8 \pi \lambda_{B} c}$ for a number density $2 c$ of a monovalent salt; $\lambda_{B}$ is given below), and $\sigma$ as the hard-core/particle diameter. Within the framework of the Derjaguin-Landau-VerweyOverbeek ("DLVO") theory, ${ }^{8}$ the contact value reads:

$$
\gamma_{c}=\frac{Z^{2}}{(1+\kappa \sigma / 2)^{2}} \frac{\lambda_{B}}{\sigma},
$$

where $Z$ is the charge of the colloids and $\lambda_{B}$ $=e^{2} / 4 \pi \varepsilon_{0} \varepsilon_{m} k_{B} T$ is the Bjerrum length of the suspending medium; $e$ is the elementary charge. These expressions will allow us to give a qualitative explanation for our observations (for a description of the theoretical assumptions that were made to predict the final equilibrium state, when the osmotic pressure difference exactly balances the electrostatic driving force, we refer the reader to Sec. I of Ref. 4). 
In a high-frequency $(1 \mathrm{MHz})$ external electric field, our particles acquire an opposing dipole moment because their dielectric constant is lower than that of the suspending medium. Consequently, they are attracted to the regions of lowest field strength ["negative dielectrophoresis (DEP)"]. ${ }^{2}$ In Ref. 4, we have shown that this is a major advantage over systems based on positive DEP-which drives the particles toward the highest field strength-as it prevents unwanted dipole-dipole interactions in the compressed suspension. Here, we used two different sample cell layouts for negative DEP. One of these was already introduced in Ref. 4, while we designed another cell especially for low ionic strength suspensions, so as to better preserve the long Debye screening length and thereby the long-ranged repulsive interactions (in our apolar solvents, the Debye screening length can easily reach several micrometers).

We kept track of the evolution of the local particle concentration and the suspension structure by means of confocal scanning laser microscopy. From what is known about the equilibrium phase behavior of soft-sphere suspensions (e.g., Ref. 9), one might expect the repulsive colloidal fluid in the electric bottle to crystallize into other lattices, besides the rhcp structure that was observed for dielectrophoretically compressed hard spheres. ${ }^{4}$ On a more basic level, though, it has not actually been tested yet how well suited an electric bottle is for the manipulation of the particle concentration in suspensions with long-ranged repulsive interactions. Therefore, we investigated for a couple of suspensions with a different range of the interparticle repulsions how the particledensity profile changed under the influence of the field gradient and how this initiated the growth of different crystal structures. Moreover, we will describe the remarkable effects that dielectrophoretic compression can have on the lattice structure, but which were not observed before for compressed hard-sphere crystals. ${ }^{4}$

\section{EXPERIMENTAL DETAILS}

\section{A. Sample cells}

We constructed an electric bottle with a "slitlike" geometry, as we described in Ref. 4 [see also Fig. 1(a) therein]. However, instead of glass slides 0 , we now used slides 00 for the spacers (thickness of $55-80 \mu \mathrm{m}, \varepsilon_{g}=6.7$ at $1 \mathrm{MHz}$; Menzel). We also designed another electric bottle using a thin borosilicate glass capillary $\left(0.1 \times 2.0 \mathrm{~mm}^{2}\right.$ inner dimensions, wall thickness of $100 \mu \mathrm{m}, \varepsilon_{g}=4.9$ at $1 \mathrm{MHz}$; Vitrocom). We covered half of its width with Scotch tape and sputter coated the other half with $3 \mathrm{~nm}$ chromium and $9 \mathrm{~nm}$ gold (Cressington, $208 \mathrm{~h}$ ). Afterwards, we removed the unwanted contact between the opposing electrodes, on the top and bottom face of the capillary, with a razor blade. The final configuration basically consisted of an approximately $1 \mathrm{~mm}$ wide parallel-plate capacitor and a $1 \mathrm{~mm}$ wide electrode-free slit next to it, running along the entire length $(\sim 5 \mathrm{~cm})$ of the capillary. The advantage of this cell is its naturally enclosed sample space; one does not need to construct side walls with spacers. We used silver paint (Jeol) and thin T2 thermocouple alloy wire (diameter of $50 \mu \mathrm{m}$, Goodfellow) for the electrical contact with the cell. Then, using Super Glue, we

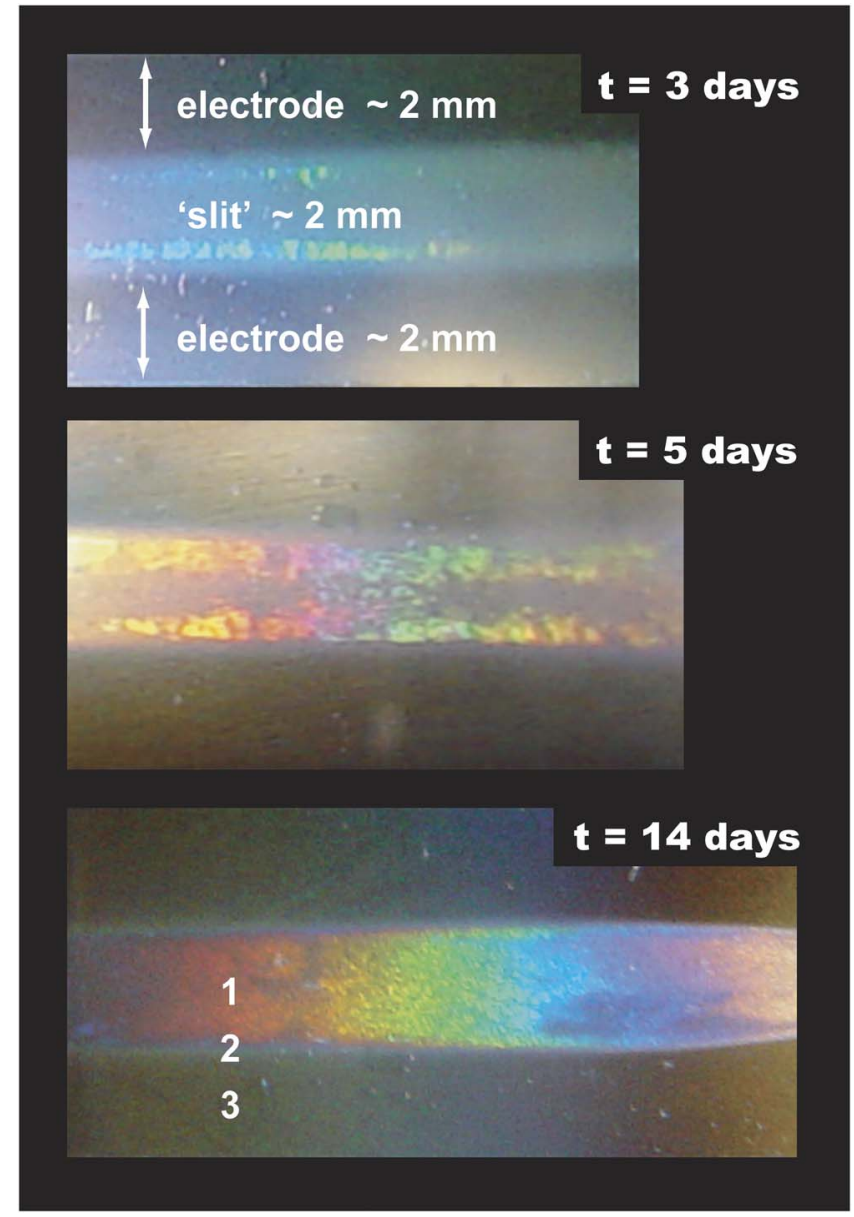

FIG. 1. (Color online) Bragg reflection images showing the crystallization induced by dielectrophoretic compression at $V_{\text {rms }}=17.7 \mathrm{~V}$ (maximum gradient of $-1.3 \times 10^{8} \mathrm{~V} \mathrm{~m}^{-2}$ ). The bright reflections clearly set the crystals apart from the more diffusively scattering colloidal fluid. The symbols in the picture at time $t=14$ days indicate the positions of the confocal images in Fig. 2. All photographs were taken in transmission, using white-light illumination.

mounted the wired capillary on top of a $1.0 \mathrm{~mm}$ thick microscope slide for extra support and ease of use on the stage of the microscope. After filling it with the colloidal suspension, the final sealing of the open ends of the cell was done with UV-curing optical adhesive (Norland, No. 68).

\section{B. Suspensions}

The suspensions consisted of PMMA spheres $\left[1.19 \mathrm{~g} / \mathrm{ml}, \varepsilon_{p} \approx 2.6\right.$ at $1 \mathrm{MHz}$ (Ref. 10)] in mixtures of as received cyclohexyl bromide (CHB, $1.33 \mathrm{~g} / \mathrm{ml}$, Fluka) and cis-decalin $(0.89 \mathrm{~g} / \mathrm{ml}$, Sigma-Aldrich) that were nearly density- and refractive-index matched. The particles were prepared by dispersion polymerization, covalently labeled with the fluorescent dye rhodamine isothiocyanate, and sterically stabilized with poly(12-hydroxystearic acid). ${ }^{11}$ The two different batches of particles used here were, respectively, 2.16 and $2.20 \mu \mathrm{m}$ in diameter, and both had a size polydispersity of $3 \%$, as determined from light scattering measurements. The dielectric constants of the solvent mixtures $\left(\varepsilon_{m}\right)$ were determined through correlation with the measured refractive indices of several mixtures and the pure CHB and cis-decalin solvents. ${ }^{12}$ We filled the slitlike sample cell with a 
suspension of the $2.20 \mu \mathrm{m}$ RITC-PMMA particles (volume fraction $\varphi=0.15$ ) in CHB with $21.01 \%$ cis-decalin by weight and the "capillary" cell with $2.16 \mu \mathrm{m}$ RITC-PMMA particles $(\varphi=0.10)$ in CHB with $27.15 \%$ cis-decalin.

\section{Electrokinetic characterization}

We estimated the Debye screening length $\kappa^{-1}$ of the suspensions by measuring the conductivity $\sigma$ of the particle-free solvent mixtures (with a Scientifica 627 conductivity meter). From the conductivity, we extracted the ionic strength $c_{i}$ using the following relation:

$$
\sigma\left[\frac{\mathrm{pS}}{\mathrm{cm}}\right]=c_{i}\left[\frac{\mathrm{mol}}{\mathrm{l}}\right] \Lambda^{0}\left[\frac{\mathrm{cm}^{2} \mathrm{~S}}{\mathrm{~mol}}\right] 10^{9}
$$

with $\Lambda^{0}$ as the limiting equivalent molar conductance of the electrolyte, here hydrobromic acid. ${ }^{13}$ We estimated $\Lambda^{0}$ for our CHB-decalin mixture using Walden's rule ${ }^{14}$

$$
\Lambda_{1}^{0} \eta_{1}=\Lambda_{2}^{0} \eta_{2}
$$

This empirical rule assumes that there is a simple Stokes relationship between an ion's mobility in the solvent of interest (1) and another reference solvent (2), for which it has been measured ( $\eta$ is the viscosity of the solvent). We determined $\Lambda_{\mathrm{H}+}{ }^{0}$ and $\Lambda_{\mathrm{Br}-}{ }^{0}$ from the known conductances of $\mathrm{HBr}$ and $\mathrm{H}^{+}$in ethanol $(\eta=1.08 \mathrm{mPa} \mathrm{s}) .{ }^{15} \mathrm{In}$ this solvent, $\Lambda_{\mathrm{HBr}}{ }^{0}$ $=88.9 \mathrm{~cm}^{2} \mathrm{~S} \mathrm{~mol}^{-1}$ and $\Lambda_{\mathrm{H}+}{ }^{0}=53.6 \mathrm{~cm}^{2} \mathrm{~S} \mathrm{~mol}^{-1}$, meaning that $\Lambda_{\mathrm{Br}-}{ }^{0}=35.3 \mathrm{~cm}^{2} \mathrm{~S} \mathrm{~mol}^{-1}$. The viscosity of our solvent mixture was $\sim 2.2 \mathrm{mPa} \mathrm{s}$ at $25{ }^{\circ} \mathrm{C}$ (measured with a Schott ViscoSystem AVS 360).

The particle charge was quantified by means of electrophoretic mobility measurements (using a Coulter Delsa 440SX) on a dilute suspension in the CHB-decalin mixture $(\varphi=0.0015)$, following the procedure developed by Kornbrekke et $a l .{ }^{16}$ We calibrated the cell with a certified conductivity standard (radiometer) to determine the cell constant, and a carboxylate-modified polystyrene mobility standard, measured in a constant current mode (Coulter EMPSL7). For our low-polar suspensions, we used the constant voltage mode with the following run parameters: $25 \mathrm{~V}, 2.0 / 0.5 \mathrm{~s}$ on/off time, and a total runtime of $60 \mathrm{~s}$. We measured the entire mobility profile (at different scattering angles), probing nine positions distributed over the entire height $(1 \mathrm{~mm})$ of the channel. Afterwards, we determined the exact position of the channel walls and the stationary layers by means of a Komagata linearization. ${ }^{17,18}$ We related the observed electrophoretic mobility to the potential at the surface of shear (the so-called "zeta" potential, $\xi$ ) using the standard electrokinetic model, implemented in the O'Brien and White MOBILITY program. ${ }^{19}$ For the suspensions we used, it is safe to assume that the plane of shear more or less coincides with the particle surface, so that the zeta potential reflects the value of the surface potential. Finally, to translate the zeta potential into a particle charge $Z$, we used the empirical relationship proposed by Loeb et al., ${ }^{17}$

$$
Z=4 \pi \varepsilon_{m} \varepsilon_{0} \frac{k_{B} T}{e} \kappa a^{2}\left[2 \sinh \left(\frac{\Psi}{2}\right)+\frac{4}{\kappa a} \tanh \left(\frac{\Psi}{4}\right)\right] .
$$

Here, $\varepsilon_{0}$ is the permittivity of the vacuum, $k_{B}$ is the Boltzmann constant, $T$ is the absolute temperature, $e$ is the elementary charge, $a$ is the particle radius, and $\Psi=\xi e / k_{B} T$.

\section{Data acquisition and analysis}

We studied our samples using confocal microscopy, either with a Yokogawa Nipkow disk system (only used to obtain single, qualitative images in a few of the experiments) or a Leica NT CSLM with numerical aperture (NA) of 1.3 $63 \times$ and NA of $1.2540 \times$ oil immersion objectives, $568 \mathrm{~nm}$ excitation, and a modified microscope stage (Rolyn, 750MS) with low-speed closed-loop motorized actuators (Newport, 850G-LS) for accurate positioning. We took data stacks between 20 and $50 \mu \mathrm{m}$ above the bottom of the sample cell and extracted the three-dimensional (3D) particle coordinates with an adapted version of the method of Crocker and Grier, ${ }^{20}$ as it was described by Royall et al. ${ }^{6}$ A single data stack consisted of $128 \times 128 \times 72$ pixels, which took around $30 \mathrm{~s}$ of scanning. The $x y$ pixels were $220 \times 220 \mathrm{~nm}^{2}$ in size, and the separation between the $x y$ slices was $440 \mathrm{~nm}$. We took these data stacks at regular distances across the entire width of the cell to obtain the complete volume fraction profile.

\section{RESULTS AND DISCUSSION}

\section{A. Dielectrophoretic compression of a soft-sphere liquid}

We started by studying the suitability of the slitlike electric bottle for compressing soft-sphere suspensions with long-ranged repulsive interactions. We first introduced this electric bottle design in Ref. 4 [see, for instance, Fig. 1(a) therein]; it basically consists of two parallel-plate capacitors of approximately $2 \mathrm{~mm}$ wide, with a $2 \mathrm{~mm}$ wide electrodefree slit in between. As the dielectric constant of our PMMA particles is lower than that of the suspending CHB-decalin mixture, they are driven toward the areas with the lowest field strength and, therefore, they collect inside the electrodefree slit (at a field frequency of $1 \mathrm{MHz}$, which prevents polarization of the double layer and unwanted electrohydrodynamic effects). We determined the exact position of the slit edges in our sample cell by microscopic inspection, giving $\pm 1044 \mu \mathrm{m}$ if we set the center of the slit to 0 . The height of the sample space enclosed by the cover slips was $90 \mu \mathrm{m}$. We measured this by scanning through with the $z$ translation stage of the confocal microscope. With these dimensions, we obtained an electric field profile that is similar to the one shown in Fig. 1(c) of Ref. 4 (taking the experimentally applied root mean squared voltage $V_{\mathrm{rms}}=17.7 \mathrm{~V}$, $\beta=\left(\varepsilon_{p}-\varepsilon_{m}\right) /\left(\varepsilon_{p}+2 \varepsilon_{m}\right)=-0.24$, and the isotropic suspension dielectric constant $\varepsilon_{s}=5.5$ (see also below and Sec. I of Ref. 4). The main features are a steep electric field gradient at the slit edges (maximum gradient of $-1.3 \times 10^{8} \mathrm{~V} \mathrm{~m}^{-2}$ ), which provides a dielectrophoretic driving force of $-3.1 \times 10^{-16} \mathrm{~N}$, and a negligible field strength inside the electrode-free slit, $\ll 0.01 \mathrm{~V} \mu \mathrm{m}^{-1}$. The latter eliminates the risk of induced 

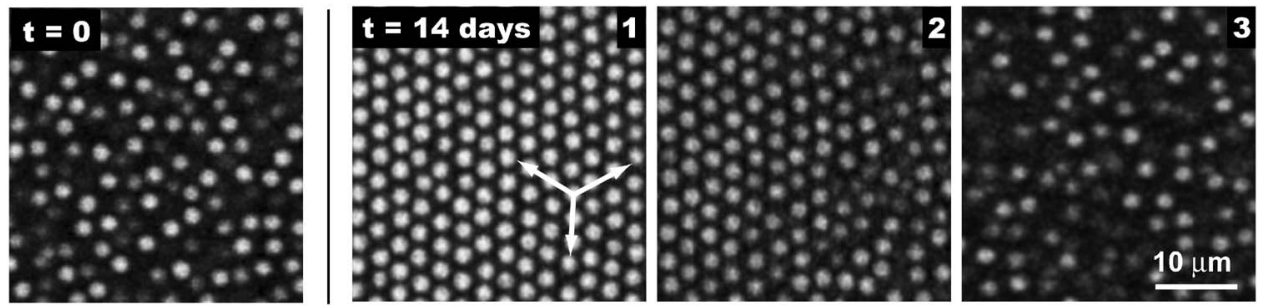

FIG. 2. Confocal microscopy images before $(t=0)$ and after 14 days of compression at $V_{\mathrm{rms}}=17.7 \mathrm{~V}$. The symbols correspond to different positions along the particle-density profile, as indicated in Fig. 1 ( $t=14$ days). The arrows indicate the three equivalent close-packed directions of the hexagonal lattice.

dipole-dipole interactions, changing the native suspension structure inside the slit. In practice, also between the electrodes, where the field was $\sim 0.05 \mathrm{~V} \mu \mathrm{m}^{-1}$, such fieldinduced effects (e.g., string formation ${ }^{5,21}$ ) were seen to be limited due to the repulsive interactions between the particles.

We filled the sample cell with a suspension of $2.20 \mu \mathrm{m}$ diameter RITC-PMMA particles in a mixture of CHB and $21.01 \mathrm{wt} \%$ decalin $\left(\varepsilon_{m}=6.1 ; \lambda_{B}=92 \AA\right)$. The overall starting volume fraction was $\varphi=0.15$, as determined by particle tracking. PMMA particles always selectively absorb a small fraction of the CHB from these mixtures. This changes both their density and polarizability. To prevent further change in the suspension during the actual dielectrophoretic compression, we let the suspension equilibrate for 10 days before turning on the electric field. From electrophoretic mobility measurements, we found the particle charge for a similar but more dilute suspension $(\varphi=0.0015)$ to be $Z=+1050 \pm 100 e$. Together with a Debye screening length $\kappa^{-1}$ $=0.80 \pm 0.3 \mu \mathrm{m}$ (corresponding to a measured conductivity of $692 \mathrm{pS} \mathrm{cm}^{-1}$ ), this gave rise to significant, long-ranged repulsive interactions between the particles. We calculate $\kappa \sigma=2.7$ and $\gamma_{c}=834[\mathrm{Eq}$. (2)], but estimate that the first could be different by about a factor of 1.5 maximum and the latter by a factor of $\sim 2$. This is due to the various assumptions needed to extract $\kappa$ and $Z$ from the electrokinetic measurements (see Sec. II).

At the start of the experiment, the entire sample was a repulsive colloidal fluid. The photographs in Fig. 1 illustrate how the electrode-free slit became fully crystalline within 14 days' time at $V_{\mathrm{rms}}=17.7 \mathrm{~V}$, while the confocal micrographs of Fig. 2 give a qualitative impression of the induced particle-density profile and the fluid-crystal interface. During the compression, the suspension between the electrodes remained a fluid, and no crystallization was observed in a similar, field-free reference cell either. Thus, the observed fluidcrystal transition was entirely due to the applied field gradient, indicating that this type of electric bottle can compress at least certain suspensions of charged particles strongly enough to push them past their phase boundaries.

From the macroscopic Bragg reflection images (Fig. 1), it seems that the crystal growth process is similar to what we have previously observed for the dielectrophoretic crystallization of hard-sphere-like suspensions. ${ }^{4}$ In that case, the particles that were transported toward the slit by dielectrophoresis were found to accumulate and crystallize at the slit edges. Newly arriving particles were added to the "outer" edges of the crystalline bands (i.e., the edges that face the electrodes). At the same time, the entire crystalline bands were seen to move in a pluglike fashion toward the center of the slit. On their way, they incorporated the particles in the central area, which added on to their "inner" edges. In this way, the crystalline bands grew inward until they met each other at the center of the slit.

With the present suspension of soft spheres, the first crystals also appeared at the slit edges within 3 days after turning on the electric field. These crystals had a packing density of $\varphi_{\text {crys }}=0.22$, which presumably was (close to) the bulk crystallization volume fraction of this suspension with long-ranged repulsive interactions (for comparison, a suspension of hard spheres displays fluid-crystal coexistence for $\varphi$ $=0.494-0.545,{ }^{1,22}$ and for the nearly hard-sphere suspension studied in Ref. 4 , we found $\varphi_{\text {crys }}=0.39-0.44$ ). The crystals were seen to grow inwards until after 14 days, the entire slit had become crystalline. At the same time, the position of the outer edges of the crystalline bands stayed nearly the same, indicating that they, indeed, displayed a continuous motion toward the center of the slit, while new particles from inside the electrodes arrived at their outer edges.

The characteristic dielectrophoretic accumulation and (heterogeneous) crystallization behavior is borne out by the evolution of the particle-density profile across the sample cell (Fig. 3) in more detail. Qualitatively, we observed the same trends as for the hard-sphere case (see, for instance, Figs. 4 and 6 of Ref. 4). First of all, within 24 h after turning on the electric field, the particle volume fraction just outside the slit dropped considerably, as these nearby particles were rapidly driven into the slit. This, in turn, caused a quick rise

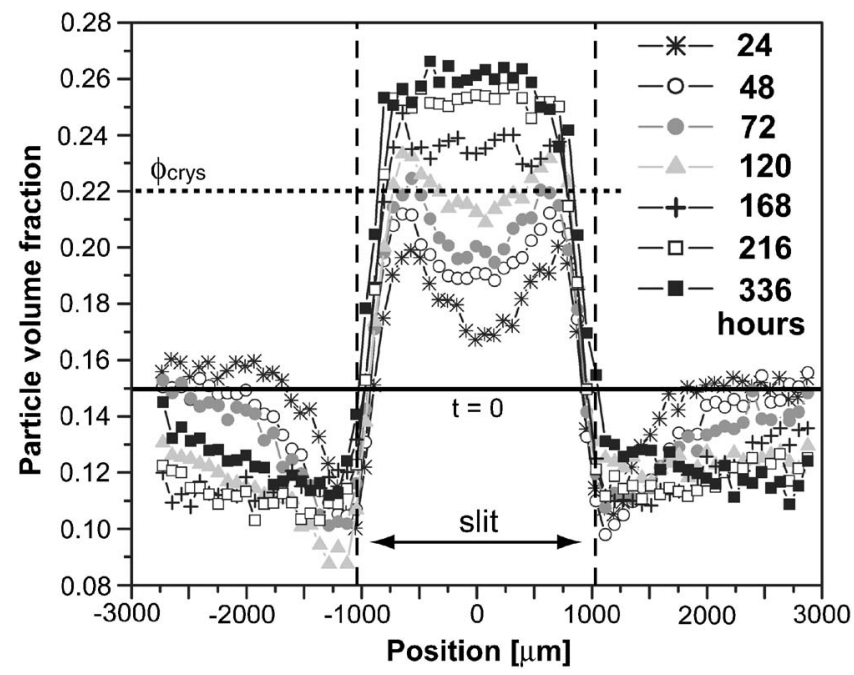

FIG. 3. Particle-density profiles across the sample cell at different times during the dielectrophoretic compression at $V_{\mathrm{rms}}=17.7 \mathrm{~V}$. The dashed lines indicate the position of the slit edges. The volume fraction of the first crystals (' $\varphi_{\text {crys }}$ '), observed after $72 \mathrm{~h}$ is also indicated (dotted line). All data were obtained from particle tracking; the estimated error was $\sim 1 \%$. 
of the particle density just inside the slit, showing up in the profile plot as two "humps" near the slit edges. The particle concentration at the center of the slit increased much more slowly. Whereas the suspension near the slit edges already crystallized after $72 \mathrm{~h}$ ( 3 days; $\varphi_{\text {crys }}=0.22$ ), it took the central area about $168 \mathrm{~h}$ (7 days) to become fully crystalline. Except for some local fluctuations, the compressed crystal then had the same density throughout, i.e., the humps disappeared.

The remarkable heterogeneous dielectrophoretic crystal growth mechanism, which appears to be essentially the same for the hard-sphere suspension studied in Ref. 4 and the repulsive soft-sphere suspension studied here, is due to a mismatch of the time scales of the different particle transport mechanisms in our electric bottles. As explained before, ${ }^{4}$ particles near the edge of the electrodes are transported relatively quickly into the slit by the dielectrophoretic force that results from the local electric field gradient. Inside the slit, however, the electric field strength quickly drops to almost zero. Thus, just after arriving inside the slit, the particles only spread further toward the center (over distances of several hundreds of micrometers) by collective diffusion without dielectrophoretic driving force, which was found to take much longer.

Looking at Fig. 3, we see that between $t=216 \mathrm{~h}$ (9 days) and $t=336 \mathrm{~h}$ (14 days) the particle-density profile did not change much anymore. We, therefore, assume that after this time the electric bottle had approached equilibrium (note that most of the compression took place far out-of-equilibrium because we instantaneously turned on a large field gradient). By then, the volume fraction of the colloidal fluid outside the slit had dropped to $\varphi \approx 0.12$, while the crystal inside the slit had been compressed to $\varphi \approx 0.26$. Note that the "dip" in the particle density just outside the slit, which was very distinct in the first $24 \mathrm{~h}$ after turning on the field, was much less pronounced after $336 \mathrm{~h}$. When the system is fully equilibrated, the particle density should be constant at constant electric field strength outside the slit because the chemical potential has to be the same throughout the entire cell (see also Sec. I of Ref. 4). However, the time to reach full equilibrium was long because the electric field gradient covered no more than $20 \%$ of the area between the electrodes. Consequently, the particles needed to travel large distances by diffusion without dielectrophoretic driving force.

In Ref. 4, we found a good agreement between the experimental and calculated particle distribution after equilibrium had been reached when we used the hard-sphere equation of state and modeled the experimental system of strongly screened charged particles $\left(\varphi_{\text {crys }}=0.39\right)$ as a suspension of hard spheres with a somewhat larger "effective" particle diameter (see Sec. I of Ref. 4). For the present suspension, however, this approach was found to be inadequate due to the pronounced softness of the particle interactions. Experimentally, the plateau value of the particle density inside the slit was lower, the volume fraction outside the slit was higher, and the density gradient at the slit edges was less steep than in the corresponding theoretical hard-sphere profile. However, also with a more sophisticated soft-sphere equation of state, we did not find agreement between the

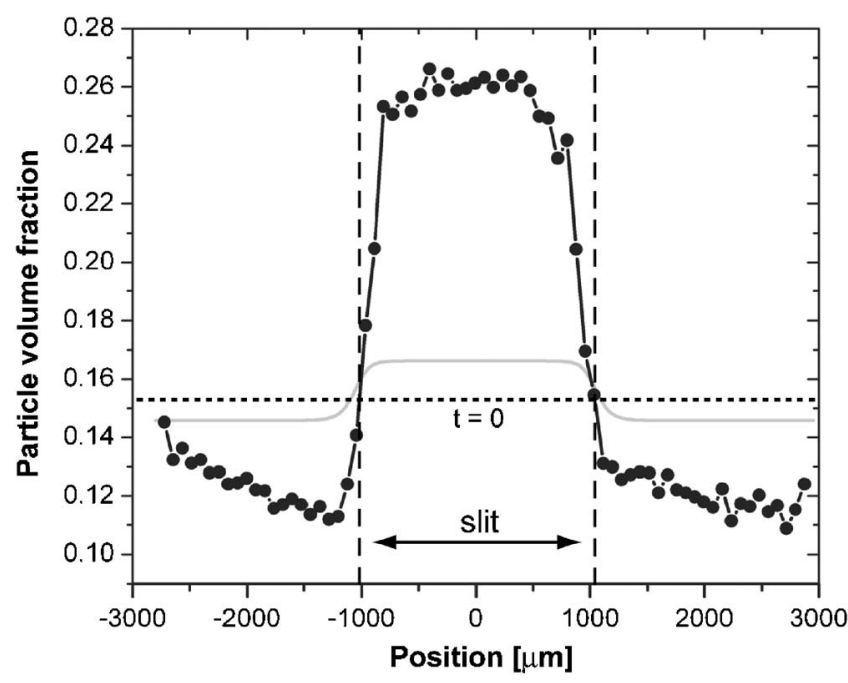

FIG. 4. Comparison of the experimental (black) and theoretically calculated (gray) particle-density profile, at $V_{\text {rms }}=17.7 \mathrm{~V}$ and using $Z=1050 e, \kappa \sigma$ $=2.7$ (see text). The dotted line indicates the initial volume fraction used in the calculation, while the vertical dashed lines indicate the positions of the slit edges.

experimental and predicted density profiles (Fig. 4). In this case, we used the Carnahan-Starling approximation for the hard-core interactions, combined with a pairwise Yukawa repulsion [as given in Eqs. (1) and (2)] and supplemented with many-body interactions. ${ }^{23}$ For our experimental parameters, $Z=1050 e$ and $\kappa \sigma=2.7$, this model indeed predicts a less steep density gradient at the slit edges, but the compression inside the slit is now strongly underestimated, with a predicted volume fraction of only $\varphi \approx 0.17$, as compared to the observed value of $\varphi \approx 0.26$. According to the theoretical equation of state, a field-induced chemical potential difference of several hundreds of $k_{B} T$ is needed to obtain the experimentally observed compression, while our electric field (at $V_{\mathrm{rms}}=17.7 \mathrm{~V}$ ) can only account for $\sim 75 k_{B} T$. At present, we do not have an explanation for the unexpectedly strong compression of our experimental soft-sphere suspensions, but it may be that our estimates of the particle charge and screening length are not precise enough or that the current theoretical model does not accurately reflect our experimental conditions. For instance, a major difference is the assumption of equilibrium between the colloidal suspension and an ion reservoir, which we do not have in our sample cells. Another possibility is a density-dependent particle charge, with a lower charge at higher volume fractions (see Ref. 7), which would facilitate compression. We are planning to test this hypothesis in a future study by analyzing the displacements of the particles around their lattice positions, so as to obtain information about the local particle interactions. $^{7,24}$

\section{B. Crystal characteristics}

Despite the similarity in growth mechanism, there are also some differences between the dielectrophoretically grown hard-sphere and soft-sphere crystals. One of these is immediately obvious from the macroscopic Bragg reflection images in Fig. 1. The first crystals that appeared after 3 days 
at the slit edges showed up as separate crystallites, with dimensions between 100 and $300 \mu \mathrm{m}$. After 5 days, these had adjusted their orientation and merged to a great extent already, forming a continuous crystalline band with a single orientation. Finally, after 14 days, the entire slit (of $\sim 2$ $\times 20 \mathrm{~mm}^{2}$ ) seemed to be filled with a single crystal that had the same orientation throughout. This is different from the hard-sphere system, where crystallites with different orientations were distinguishable as separately reflecting entities at all times (see Fig. 2 of Ref. 4). A closer inspection with confocal microscopy indeed confirmed that the soft spheres essentially formed a single crystal. In some places, often near an anomously large inclusion, the lattice was slightly curved though, causing a small change in orientation (typically $<10^{\circ}$ ). It must be due to the soft repulsive interactions that the initial crystallites so easily adjusted their different orientations and seamlessly merged together. Note that the repulsive interactions keep the particles from touching each other in the final crystalline packing of Fig. 2.

Interestingly, the close-packed directions of the nearly perfect hexagonal layers (indicated in Fig. 2) followed the slit edges even more closely than was the case in the hardsphere system. ${ }^{4}$ Because there are three equivalent directions with a $60^{\circ}$ difference, the maximum possible angle with respect to the slit edge is $30^{\circ}$. Experimentally, however, most of the crystal formed a much smaller angle $\left(<5^{\circ}\right)$. Only occasionally, in places where the lattice was locally curved, we observed angles up to $15^{\circ}$. Just like in the hard-sphere case, we hypothesize that shear effects possibly induce an alignment of the crystals during the early nucleation and growth stages. ${ }^{25}$ It is remarkable, though, that this produced such large and well-oriented crystals. The fact that the closepacked hexagonal layers ran parallel to the bounding cover slips indicates that the structure was the result of heterogeneous crystal growth, which facilitates at least partial alignment of the crystal. Using the "overall stacking parameter" $\alpha$, as defined in Ref. 4, we characterized the stacking sequence of the final crystal. For a perfect face-centered-cubic (fcc or $A B C$ ) stacking, $\alpha=1$, for a perfect hexagonal closepacked (hcp or $A B A B$ ) stacking, $\alpha=0$, and for more random sequences (rhcp or random hexagonal close-packed) it assumes intermediate values. ${ }^{26}$ We analyzed 15 stacks of around 25 layers thick and found that $\alpha=0.59$ on average. We did not observe any values smaller than 0.38 , nor ones larger than 0.77 . Thus, there apparently was no preference for one stacking sequence over another, giving rise to an almost rhcp structure. This is in agreement with the observation that for a considerable range of parameters, the free energy between fcc and hcp is smaller than $0.01 k_{B} T$ for particles with a screened Coulomb potential. ${ }^{27}$

The phase behavior of charged particles, interacting via a DLVO-type screened Coulomb pair potential [Eqs. (1) and (2)], has been extensively studied in computer simulations. ${ }^{9,27,28}$ Depending on the strength and the range of the repulsive interactions, the soft-sphere phase diagram contains two stable crystalline phases: The fcc/rhcp structure and a body-centered-cubic (bcc) crystal structure. The latter only occurs for sufficiently high contact values $\left(\gamma_{c} \gtrsim 8\right)$, in combination with a long Debye screening length (roughly $\kappa \sigma$ $\lesssim 5$ for $\left.8 \leqslant \gamma_{c} \leqslant 81\right) .{ }^{9}$ No results have been reported for contact values as high as ours $\left(\gamma_{c}=834\right)$, but it was shown that for $\gamma_{c} \geqslant 20$, the behavior is well described by the phase boundaries of point-Yukawa particles. If we map for our experimental parameters the point-Yukawa system onto a hardcore Yukawa system, the fluid-bcc-fcc/rhcp triple point is found at $\kappa \sigma=2.3$. The experimental observation of a fluidrhcp transition at $\kappa \sigma=2.7$ is in qualitative agreement with this.

Although the present experiment was not specifically set up for it, we also studied the melting process of the crystal after releasing the compressive electric field. Just like for the hard-sphere crystal in Ref. 4, melting started at the outer edges of the crystal, giving the fluid-crystal interface a rather rough and disordered appearance, with small crystallites breaking away from the main body of the crystal. However, whereas the hard-sphere crystal needed 2 months to fully melt back into a colloidal liquid, this took only 11 days for the present soft-sphere crystal. In fact, a significant fraction of the crystal $(>35 \%)$ disappeared within 2 days already, but at later times, the melting process slowed down. Nevertheless, it is likely that the long-ranged repulsive particle interactions speed up the melting process considerably, even though at present it is not clear if gravity played a role as well, as we observed slight sedimentation of the particles during the melting process. Also, on a qualitative level, the evolution of the particle-density profile was quite different. In Ref. 4, the hard-sphere crystal was seen to relax to the packing density at which the first crystals appeared during the preceding compression step. After this initial drop, the volume fraction of the shrinking crystal remained nearly constant. For the present soft-sphere suspension, it looked more as if the "high density" plateau of the profile was continuously broadening. This reduced the crystal packing density, but as soon as it reached the original crystallization volume fraction $\left(\varphi_{\text {crys }}=0.22\right)$, the entire crystal quickly melted. The steady decrease in packing density indicates an expansion of the crystalline lattice, which indeed was visible in our confocal microscopy images as an increasing interparticle spacing.

\section{Compressing softer spheres: Liquid-bcc transition}

As explained above, soft spheres potentially offer a richer phase behavior than hard-sphere suspensions, with the existence of a body-centered-cubic phase for sufficiently long-ranged repulsive interactions. To investigate the accessibility of the bcc phase in our electric bottles, we used a suspension with a longer screening length, together with a different cell design that can better preserve the long-ranged repulsive interactions, while providing enough compression. The suspension consisted of $2.16 \mu \mathrm{m}$ diameter RITCPMMA particles in a mixture of cyclohexyl bromide and $27.15 \mathrm{wt} \%$ decalin $\left(\varepsilon_{m}=5.6 ; \lambda_{B}=10 \mathrm{~nm}\right)$. The conductivity of this solvent mixture was only $122 \mathrm{pS} \mathrm{cm}^{-1}$, giving rise to a long Debye screening length of $1.8 \pm 0.8 \mu \mathrm{m}$, or 0.8 $\leqslant(\kappa \sigma=1.2) \leqslant 2.2$. The overall starting volume fraction of the particles was $\varphi=0.10$. From electrophoretic mobility measurements on a similar but more dilute suspension $(\varphi$ 
(a)

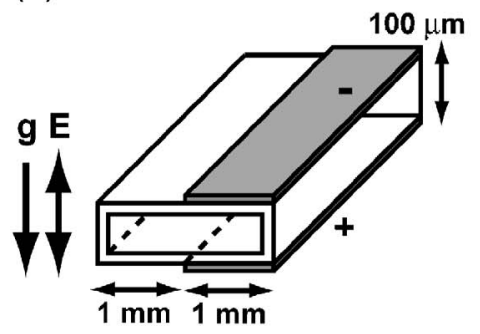

(b)

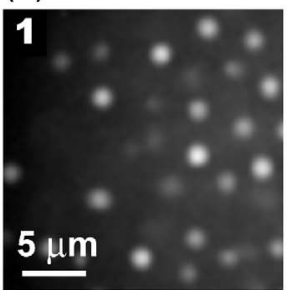

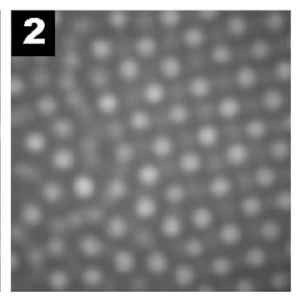
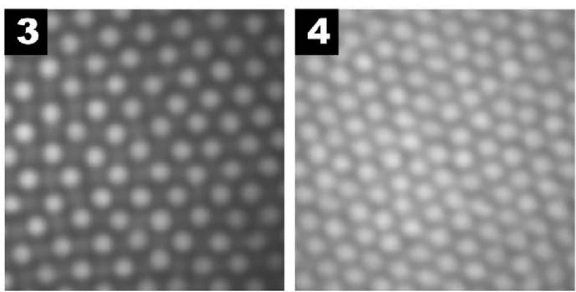

FIG. 5. (a) Schematic drawing of a glass-capillary based electric bottle. The respective directions of gravity and the (ac) electric field when the cell was mounted on top of the microscope are also indicated. The thickness of the glass (white) and the electrodes (gray) is not drawn to scale. (b) Confocal microscopy images of a soft-sphere suspension in the electric bottle of panel (a), after compression for $336 \mathrm{~h}$ at $V_{\text {rms }}=21.2 \mathrm{~V}$. The numbers correspond to different positions along the particle-density profile, which approximately were at the slit edge (1) and $200 \mu \mathrm{m}(2), 500 \mu \mathrm{m}(3)$ and $800 \mu \mathrm{m}(4)$ into the electrode-free slit.

$=0.0015)$, we found the particle charge to be $Z=+570 \pm 60 e$. Equation (2) then gives a contact value of $\gamma_{c}=587$, but this could be different by about a factor of 2 maximum.

The electric bottle differed from the earlier layout, as we used a thin glass capillary to construct the sample space, instead of separate cover slips and glue [Fig. 5(a)]. The basic idea was still the same, however. By depositing electrodes parallel to the length of the capillary $(\sim 5 \mathrm{~cm})$, we created a $1 \mathrm{~mm}$ wide parallel-plate capacitor and a $1 \mathrm{~mm}$ wide electrode-free slit next to it (this means that particles now entered the slit from one side only). According to the specifications of the capillary, the sample space was $\sim 100 \mu \mathrm{m}$ high and the walls $\sim 100 \mu \mathrm{m}$ thick. Obviously, this design is less sensitive to possible contamination and, thereby, is more efficient at conserving the long screening length of the low ionic strength suspension inside.

At the start of the experiment, the entire sample was a colloidal fluid with clear long-ranged repulsive interactions. Figure 5(b) qualitatively shows the particle-density gradient which resulted after compressing this suspension for $336 \mathrm{~h}$ (14 days) at $V_{\text {rms }}=21.2 \mathrm{~V}$. At this time, there were no longer any particles between the electrodes, they had all accumulated inside the electrode-free slit. Near the slit edge was a small stretch of fluid with a quickly increasing density. Further inward $(\sim 200 \mu \mathrm{m})$, the suspension crystallized and then remained crystalline throughout the rest of the slit. Scanning through, from the bottom to the top, we found the 3D crystal structure to be bcc everywhere. Close to the fluid-crystal interface, the (100) face of this structure was oriented parallel to the bounding walls, giving the lattice a "square" appearance [e.g., snapshot 3 in Fig. 5(b)]. Deeper inside the slit, at higher particle densities, the crystal changed its orientation, so that the (110) face, which is the closest packed plane for this crystal structure, ran parallel to the wall (snapshot 4). As expected for a bcc structure, these (110) layers had a mildly distorted hexagonal appearance and were stacked in the characteristic $A B A B$ sequence. The observation of a bcc structure (for $\kappa \sigma=1.2$ and $\gamma_{c}=587$ ) is in accordance with the phase behavior seen in computer simulations, which predict the fluid-bcc-fcc/rhcp triple point to occur at $\kappa \sigma=2.4$ for $\gamma_{c}=587$. Note, however, that the pointYukawa mapping gives $\varphi_{\text {fluid-bcc }} \approx 0.01$, while in reality, the suspension only crystallized at $\varphi_{\text {crys }} \approx 0.15$ (see below).
This discrepancy may be due to the fact that, in general, at low $\kappa \sigma$, the fluid-bcc coexistence turns to higher packing fractions again, but at this low ionic strength, we cannot exclude possible charge regulation effects either (see Ref. 7). In future work, we will investigate this further by analyzing the displacements of the particles around their lattice positions, which provides information about the interaction potential. $^{7,24}$

Knowing the crystal structure, we estimated the local particle volume fraction from the observed interparticle spacing in the lattice. In this way, we found $\varphi \approx 0.15$ close to the fluid-crystal interface, and $\varphi=0.28$ near the bounding side wall. For the complete gradient $0 \leqslant \varphi \leqslant 0.28$, as the density of the fluid dropped all the way to zero. Apparently, this capillary electric bottle compressed suspensions very efficiently too, just like the electric bottle made out of cover slips. However, the density gradient now was less steep, probably due to the softer, longer-ranged repulsions between the particles. We point out that if one can achieve sufficient compression, it should be possible to observe both a fluidbcc and a subsequent bcc-fcc/rhcp transition in a single particle-density gradient because at sufficiently high packing fractions, there always exists an fcc/rhcp phase which is favored by the hard-core repulsion of the particles.

\section{Crystal buckling}

As we mentioned before, the suspension that crystallized into a rhcp structure (Figs. 1 and 2) reached a nearly stable particle distribution throughout the sample cell after $336 \mathrm{~h}$ (14 days) of compression at $V_{\mathrm{rms}}=17.7 \mathrm{~V}$ (Fig. 3). After that, the packing density of the crystal could still be raised further, though by continued compression at a higher field strength. During $168 \mathrm{~h}$ (7 days) at $V_{\mathrm{rms}}=35.4 \mathrm{~V}$ (maximum gradient of $-2.7 \times 10^{8} \mathrm{~V} \mathrm{~m}^{-2}$ ), the crystal compacted significantly from $\varphi=0.26$ to $\varphi=0.31$. At this field strength, the dielectrophoretic driving force was $\sim 4.5 \times$ larger than before, while the field inside the electrode-free slit was still negligible. However, now there was a noticeable effect of the fieldinduced dipole interactions on the suspension structure inside

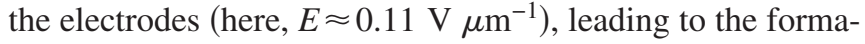
tion of short "strings" of particles and a slowdown of particle transport.

Upon further compression, the close-packed planes of 

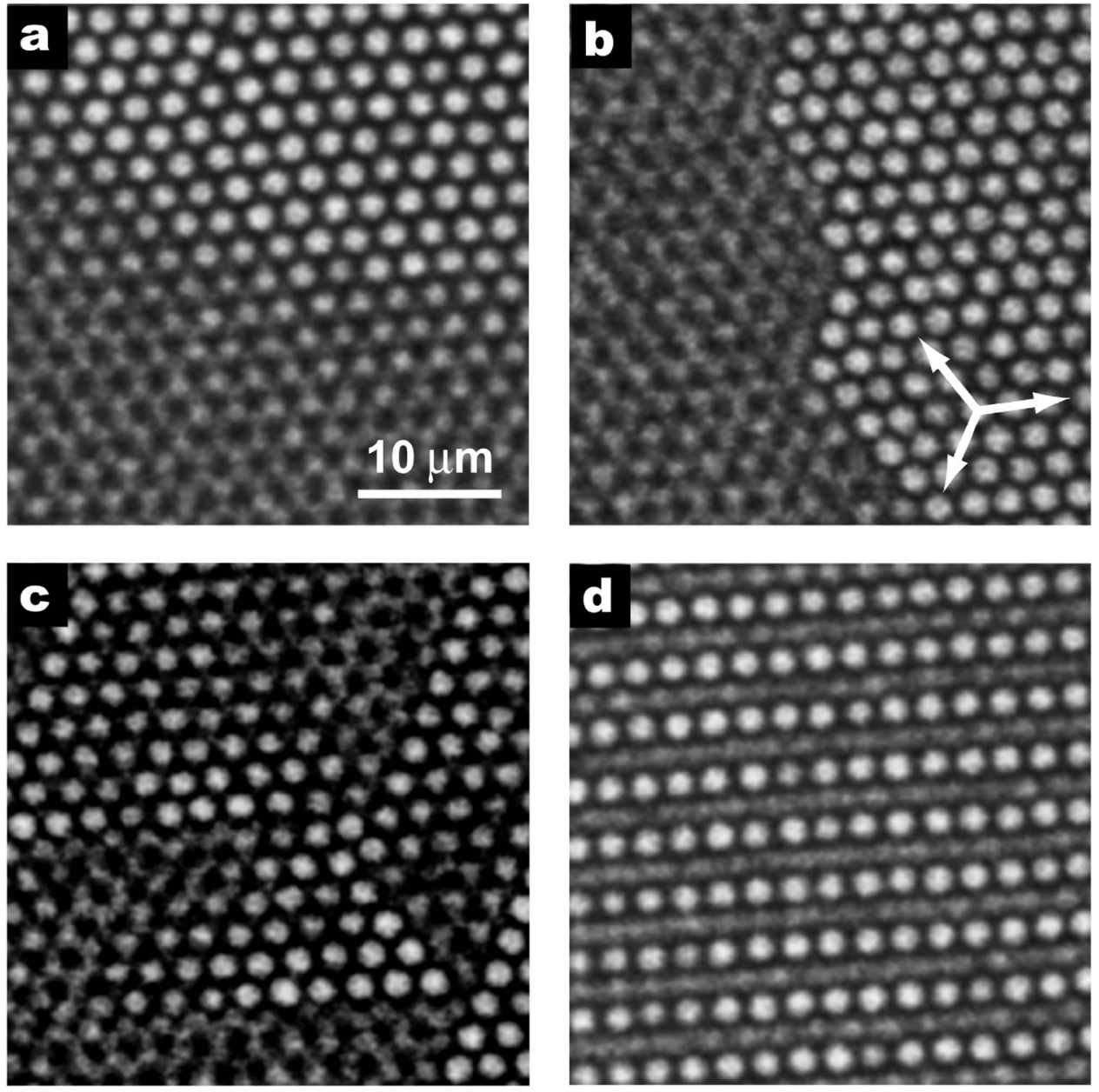

FIG. 6. Confocal microscopy images of different buckling patterns, which appeared after continued compression of the soft-sphere crystal of Figs. 1 and $2\left(168 \mathrm{~h}\right.$ at $V_{\mathrm{rms}}=35.4 \mathrm{~V}$, maximum gradient of $-2.7 \times 10^{8} \mathrm{~V} \mathrm{~m}^{-2}$ ). The arrows in (b) indicate the three equivalent close-packed directions of the hexagonal lattice. The slit edges ran parallel to the top and bottom sides of the images. the rhcp structure became denser and overall the hexagonal order improved because the particle motion around the ideal lattice positions became more confined. However, in a number of places we locally observed a new kind of lattice defect/distortion which was not present before at the lower electric field strength: Buckling. Figure 6 shows a few examples of the different instances of buckling that were observed. We only show images of single crystal planes, but these distortion effects were 3D phenomena. All the planes above and below, about 45 in total, displayed the same pattern, although with a varying degree of pronouncedness. In all cases, the buckling involved a shift of (a part of) the hexagonal planes in the ' $z$ direction,' the direction normal to the bounding top and bottom cover slips.

In Fig. 6(a), the buckling occurred on a rather long length scale. The hexagonal plane was seen to gradually curve out of focus in the lower half of the field of view. Further away, outside the field of view, it curved back again, and this "wave" then repeated itself a few times. This buckling pattern was different from the situations shown in Figs. 6(b) and 6(c). Instead of a smooth transition, the latter displayed a very sudden jump and the patches that were shifted in the $z$ direction were limited in size. The distinct edge was seen to always follow the close-packed directions of the hexagonal lattice, giving rise to a zigzag [Fig. 6(b)] or a triangular appearance [Fig. 6(c)]. The most intriguing buckling pattern, however, is shown in Fig. 6(d). Here, individual par- ticle rows, which ran nearly parallel to the slit edges, shifted out of the originally hexagonal plane. We observed this pattern in a, by approximation, rectangular patch of about 400 $\times 200 \mu \mathrm{m}^{2}$, with the longest side oriented parallel to the slit edges. Just as in the other cases, this buckling pattern was fully three dimensional, spanning almost the entire height of the sample space.

Figures 7(a) and 7(b) show the stacking of the buckled particle layers along the $z$ direction, in a rendering of the three-dimensional particle coordinates of a small part of the buckled domain in Fig. 6(d) ( 1600 particles in total). The first six layers (in white) were close to the bottom wall and were nearly undistorted (same near the top wall, not shown). All the layers in between (darker spheres) were buckled, with an increasing "amplitude" deeper inside the sample. The black and gray colors identify the particles that originally belonged to the same hexagonal particle plane, while the shading highlights how particle rows were squeezed out of these originally flat planes. Together, the particles made up a unique four-layer unit, which repeated itself eight times (not all repeats are shown). In another rendered representation, now of $\sim 1700$ particles at one of the boundaries of the buckled domain, Fig. 7(c) shows how the particle planes in the buckled patch were seamlessly connected with the surrounding, undistorted lattice. Again, the white spheres in these images constitute the first few undistorted layers, and black and gray identify the partially buckled particle planes. The 
(a)

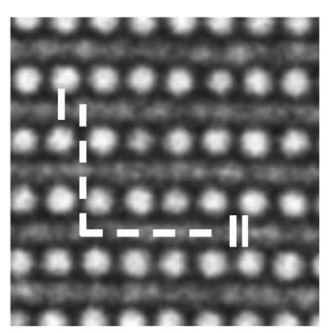

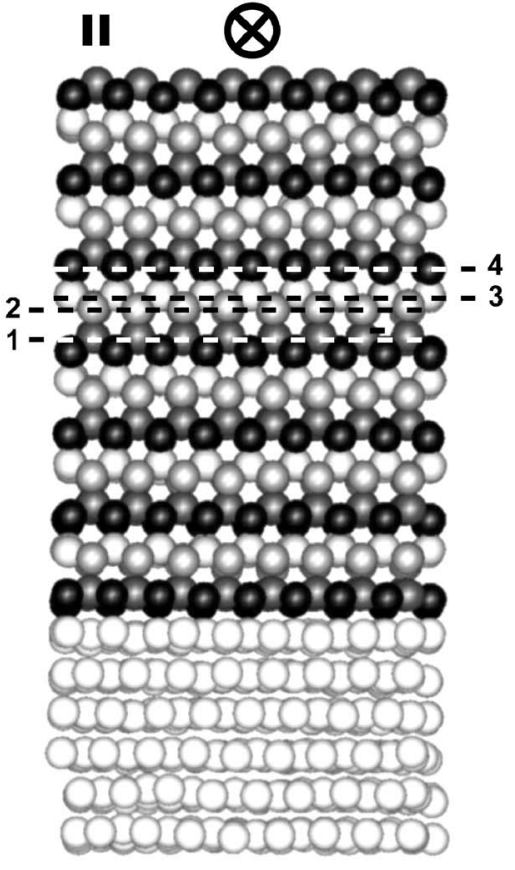

(c)

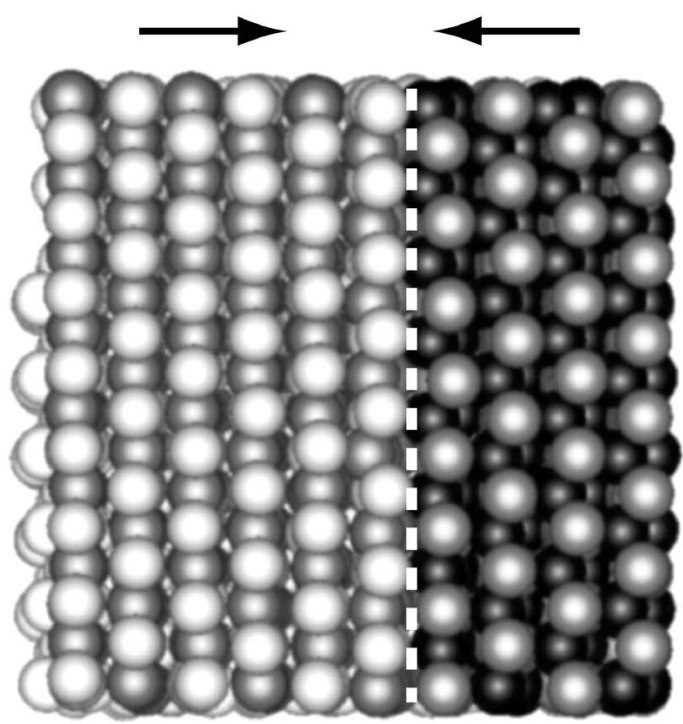

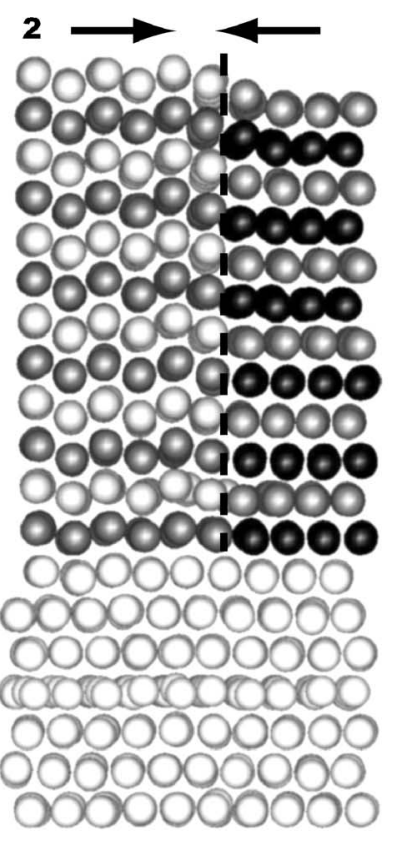

FIG. 7. Rendering of experimentally determined particle coordinates revealing the three-dimensional structure of the buckled domain in Fig. 6(d). The radius of the model spheres reflects the real size of the particles. In all cases, the direction in which the crystal experienced the compressive dielectrophoretic force is indicated with arrow (tail)s. (a) Lines I and II indicate the two different cuts through the lattice that are represented in panel (b). (b) Rendering of the buckled domain, showing the stacking of the layers in the $z$ direction. The white spheres constitute the (nearly) undistorted hexagonal particle planes at the bottom wall, whereas the darker spheres represent the buckled layers higher up. The indices in II highlight the four-layer repeat unit. (c) Rendering of the transition between the buckled domain (left, light spheres) and the neighboring, undistorted hexagonal lattice (right, dark spheres). Panel (1) is a "top view," showing a superposition of the different particle layers. The side view in (2) is rotated by $90^{\circ}$ with respect to (1), and shows the stacking of the layers in the $z$ direction. The dashed lines highlight the approximate transition between the buckled and undistorted parts of the lattice. shading now indicates the buckled (light) and undistorted (dark) parts of these layers. Note that in the buckled part the distorted particle planes effectively assume a "bridge-site" stacking, instead of the mixed $A B A / A B C$ stacking of the original hexagonal planes [Figs. 7(c1)].

Buckled structures have been seen before in experiments on strongly confined colloidal systems. In 1983, Pieranski et al. reported a characteristic sequence of structures with triangular $(\triangle)$ and square $(\square)$ symmetries, observed for hard spheres confined to a wedge shaped cell: $n \triangle \rightarrow(n+1) \square$ $\rightarrow(n+1) \triangle$ for increasing gap size. ${ }^{29}$ The sequence is due to a competition between two packing factors: Spheres pack more efficiently in a triangular arrangement, but the height of a stack of $n$ square layers is smaller. What is more interesting in light of our experimental observations, though, is what happens when the gap is too large for the $n \triangle$ structure but too small for the $(n+1) \square$ structure. In that case, different intervening structures become stable at sufficiently high density, e.g., prism, rhombic, and buckled phases. Some of these phases were recently observed in experiments ${ }^{30}$ and many more were predicted by Fortini et al. in computer simulations of hard spheres confined between two parallel hard walls. ${ }^{31}$ We studied charged spheres, instead of hard spheres, and a much larger wall separation, of about 40 particle diameters, instead of 1-5 diameters. Nevertheless, the buckled structure we observed [Fig. 6(d)] corresponds to Fortini's so-called $2 \mathcal{B}$ buckled phase, except that in our case it consisted of 32 layers in total, instead of just 2 . In the small system-size, hard-sphere phase diagram, the $2 \mathcal{B}$ phase interpolates between the $1 \triangle$ and $2 \square$ phases. Basically, the $1 \triangle$ phase is split up into two sublayers consisting of particle rows that are displaced in height, just like we saw in Fig. 7. 
(a)

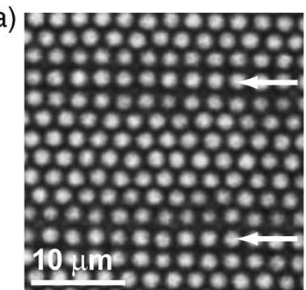

(b)

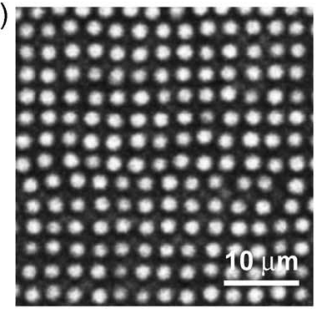

1
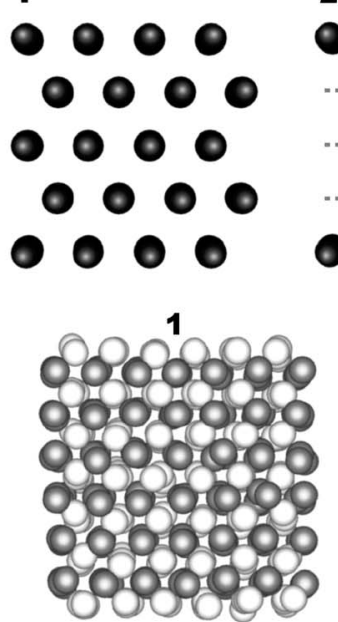

2

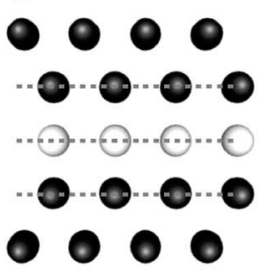

3

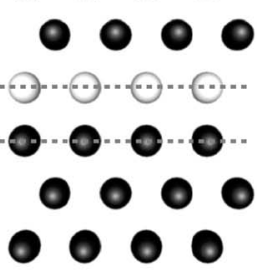

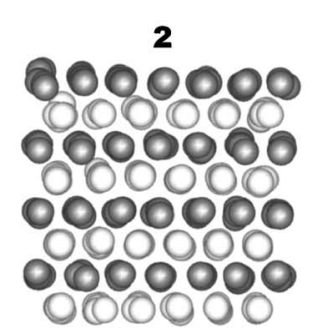

FIG. 8. Relaxation of the previously buckled domain $48 \mathrm{~h}$ (a) and $264 \mathrm{~h}$ (b) after switching off the compressive electric field ( $V_{\text {rms }}=0$ ). (a) Confocal micrograph inside the bulk with arrows indicating the locations of non-alternating particle rows. Panels (1)-(3) present schematic drawings of one of the previously buckled particle layers after relaxation. In (1) all layers went back to the original hexagonal packing, but in (2) and (3), a particle row of a layer above or below was incorporated. In (2), the inserted particles (white) pushed out a row of the original hexagonal plane; in (3), the additional row was inserted without pushing out other particles. The dashed lines indicate the rows that together form a square symmetry. (b) Confocal micrograph inside the bulk after $264 \mathrm{~h}$ at zero field. Panels (1) and (2) show the rendered coordinates of the particles in this domain, revealing the three-dimensional structure. The radius of the model spheres reflects the real size of the particles. Representation (1) shows the structure as it was seen in the confocal image of (b) (top view); (2) is the same structure $90^{\circ}$ rotated ("side view"). The particle layers along the $z$ direction were alternatingly colored gray and white [see (2)] to facilitate recognition of the different $z$ layers in (1). Note: The slit edges ran parallel to the top and bottom sides of the confocal images.

Naturally, the particle concentration is one of the parameters that determine the phase behavior of colloids. However, the buckled domain coexisted with undistorted hexagonal lattice, while we found no difference in packing density between these two neighboring areas (both had $\varphi=0.33$ ). Of course, the wall separation can be another control parameter for the phase behavior (see Fig. 2 of Ref. 31). It is unlikely that in our experiments, confinement effects determined the observed phase behavior, though, because the wall spacing was relatively large, making possible differences in packing efficiency very small. Instead, the buckling was probably due to the anisotropic nature of the dielectrophoretic compression in combination with an anisotropic response of the crystals for certain orientations. Qualitatively, a similar distortion is observed if one tries to compress a two-dimensional (2D), close-packed array of balls in a direction perpendicular to one of the close-packed lines. In that case, individual rows of particles move upward, out of the $2 \mathrm{D}$ plane, in a similar fashion as we observed here in three dimensions. Interestingly, we did not observe any buckling effects for the bcc crystal described above, which may not have been compressed enough for that, or the dielectrophoretically compressed suspension of nearly hard spheres in Ref. 4. Also, Sullivan et al. did not report about it for their hard-sphere suspensions. ${ }^{3}$ The absence of compression-induced buckling in hard-sphere crystals is likely due to the larger elastic moduli of these denser crystals. To first order approximation, the strength of a crystal, as given by its elastic moduli, is proportional to the density of bonds that a particle has with its nearest neighbors. This even holds for hard-sphere crystals, where the only contribution to the stability of the crystal is entropic. ${ }^{32}$ Thus, colloidal crystals, which consist of the same particles but that have a decreased lattice constant (as is the case for the hard-sphere-like crystals in Ref. 4, in com- parison to the present soft-sphere crystal), will be $y^{3}$ times stronger, where $y$ is the ratio of the lattice constants of the soft- and the hard-sphere crystals. It is important to note that the crystal structure and the stacking disorder were the same for the hard-sphere and soft-sphere crystals, which suggests that this did not cause the difference in buckling behavior. At present, we do not have a full understanding of all the differences in the behavior of compressed hard- and soft-sphere crystals, but we will investigate this further in future experiments and theoretical modeling.

Finally, we will describe another interesting change in the soft-sphere crystal structure, which occurred when we let the buckled structure of Fig. 6(d) relax again (by switching off the electric field, $\left.V_{\text {rms }}=0 \mathrm{~V}\right)$. After 2 days $(48 \mathrm{~h})$ at zero field, all particle layers had more or less relaxed back to their original hexagonal packing, but they were not completely flat yet [Fig. 8(a)]. Moreover, they contained a considerable number of defects, which consisted of rows of particles whose positions were not shifted with respect to the neighboring rows in the same plane. In the confocal microscopy images, these "nonalternating" rows showed up as narrow bands with a square symmetry, running parallel to the slit edges. We envision that such a square structure could come about in one of the two ways depicted in Figs. 8(a1)-8(a3). In both cases, a particle row that was "squeezed" out of the plane during the buckling process does not return to the layer from which it originated, but goes to the neighboring layer instead (i.e., in Fig. 7, a "gray" particle row moving over to the adjoining "black" layer upon relaxation). If these additional particles, in turn, push out one of the particle rows of the neighboring plane, one ends up with a "square band" of three particle rows wide [Fig. 8(a2)]. If the incorporation does not lead to the removal of another particle row from that plane, the result is a square band of two rows wide [Fig. 
8(a3)]. Experimentally, we observed the three-row bands more frequently than the twofold counterpart, forming a 3D defect structure in which the narrow square bands were effectively being passed on from layer to layer.

Over time, the square bands were seen to broaden and eventually, after 11 days (264 h) at zero field, the stacking of (defective) hexagonal layers in the previously buckled domain had completely disappeared. Instead, we observed extensive areas of square lattice, starting directly at the bottom wall and ending at the top wall [Fig. 8(b)]. A rendering of the 3D particle coordinates reveals that this was the (100) plane of a face-centered-cubic structure [Figs. 8(b1) and 8(a2)]. Apparently, it is possible to switch the original rhcp structure, with the hexagonal (111) planes parallel to the bounding cover slips, via a buckled phase, to a differently oriented fcc crystal. Note that face-centered cubic is the structure with the lowest free energy, but that the less densely packed (100) surface has a higher surface free energy than the (111) surface. Further analysis is required to identify the reasons for this remarkable change in structure, and to find out whether gravity plays a role in the relaxation process. Also, the fact that relaxation took place far out-of-equilibrium, because we switched off the field instantaneously, could be an important factor.

\section{CONCLUSIONS}

In Refs. 3 and 4, it was shown that dielectrophoretic compression provides excellent control over the colloid concentration in hard-sphere suspensions. Here, we have demonstrated that it is also a suitable technique for the manipulation of suspensions of charged particles ("soft spheres"), with long-ranged repulsive interactions. In particular, we obtained sufficient compression to push such suspensions past their (fluid-crystal) phase boundaries. The results reported here and in Ref. 4 suggest that electric bottles should be applicable to a diversity of soft matter systems with different particle interactions, providing access to widely varying (phase) behavior.

With different soft-sphere suspensions and different sample cell designs, all tailored for the accumulation of particles in a field-free zone by negative DEP, we obtained both a fluid-rhcp and a fluid-bcc transition. This is in qualitative agreement with the expected equilibrium phase behavior. The rhcp crystals were seen to grow by the same mechanism, as what we have previously reported for the dielectrophoretic crystallization of hard spheres. ${ }^{4}$ However, the compressed soft-sphere suspensions also displayed some distinct behavior, which was not seen for the hard-sphere systems. First of all, the soft spheres formed remarkably large single crystals, covering the entire field-free slit of several millimeter wide and a couple of centimeter long. Thus, the electric bottle layout should provide an easy way to exactly set the final size and shape of these soft crystals. Second, we discovered that it is possible to compress the soft-sphere crystal so strongly that it experiences an anisotropic deformation, showing up as a buckling of the lattice. This is interesting as a model system, e.g., to study crystals under stress because the exact compression and relaxation rates can be precisely controlled. Moreover, it could be used to create different lattice structures. For instance, we observed that a strong compression followed by a full relaxation (taking place far outof-equilibrium) can reproducibly transform a (111) oriented rhcp structure into a (100) oriented fcc crystal.

Finally, from a more fundamental perspective, one could use electric bottles as a convenient test for the different theoretical models of soft-sphere interactions (e.g., pair potentials versus many-body effects), besides other things. In the present study, we did not obtain quantitative agreement between the experimental and the predicted density profiles, but in future work, we should be able to improve on this with more precise determinations of the suspension properties (particle charge, screening length, and possible volume fraction dependencies) and measurements of the elastic moduli of the crystals. $7,24,33$

\section{ACKNOWLEDGMENTS}

We thank M. T. Sullivan for many useful discussions, D. Derks for synthesis of the $2.16 \mu \mathrm{m}$ particles, and H. D. Goldbach for the ITO deposition. B. Zoetekouw and R. van Roij are acknowledged for computing the theoretical softsphere equation of state. Finally, we appreciate the efforts of P. M. Chaikin, A. D. Hollingsworth, and W. B. Russel to facilitate the stay of M.E.L. at the Princeton Institute for the Science and Technology of Materials during the start-up of this research. This work was supported by the Stichting voor Fundamenteel Onderzoek der Materie and the Nederlandse Organisatie voor Wetenschappelijk Onderzoek.

${ }^{1}$ P. N. Pusey and W. van Megen, Nature (London) 320, 340 (1986).

${ }^{2}$ H. A. Pohl, Dielectrophoresis: The Behavior of Neutral Matter in NonUniform Electric Fields (Cambridge University Press, Cambridge, 1978).

${ }^{3}$ M. T. Sullivan, K. Zhao, A. D. Hollingsworth, R. H. Austin, W. B. Russel, and P. M. Chaikin, Phys. Rev. Lett. 96, 015703 (2006).

${ }^{4}$ M. E. Leunissen, M. T. Sullivan, P. M. Chaikin, and A. van Blaaderen, J. Chem. Phys. 128, 164508 (2008).

${ }^{5}$ A. Yethiraj and A. van Blaaderen, Nature (London) 421, 513 (2003).

${ }^{6}$ C. P. Royall, M. E. Leunissen, and A. van Blaaderen, J. Phys.: Condens. Matter 15, S3581 (2003).

${ }^{7}$ C. P. Royall, M. E. Leunissen, A.-P. Hynninen, M. Dijkstra, and A. van Blaaderen, J. Chem. Phys. 124, 244706 (2006).

${ }^{8}$ B. Derjaguin and L. Landau, Acta Physicochim. URSS 14, 633 (1941); E. J. Verwey and J. T. Overbeek, Theory of the stability of lyophobic colloids (Elsevier, New York, 1948).

${ }^{9}$ A.-P. Hynninen and M. Dijkstra, Phys. Rev. E 68, 021407 (2003).

${ }^{10}$ G. P. Mikhailov and T. I. Borisova, Polym. Sci. U.S.S.R. 2, 387 (1961).

${ }^{11}$ G. Bosma, C. Pathmamanoharan, E. H. A. de Hoog, W. K. Kegel, A. van Blaaderen, and H. N. W. Lekkerkerker, J. Colloid Interface Sci. 245, 292 (2002).

${ }^{12}$ M. E. Leunissen, Ph.D. thesis, Utrecht University, 2007 (available at http://www.colloid.nl)

${ }^{13}$ A. D. Hollingsworth, M. E. Leunissen, A. Yethiraj, A. van Blaaderen, P. M. Chaikin, and W. B. Russel (to be published).

${ }^{14}$ P. Walden, Z. Phys. Chem. 55, 207 (1906); 55, 246 (1906); R. M. Fuoss, J. Am. Chem. Soc. 80, 5059 (1958).

${ }^{15}$ G. J. Janz and S. S. Danyluk, Chem. Rev. (Washington, D.C.) 60, 209 (1960).

${ }^{16}$ R. E. Kornbrekke, I. D. Morrison, and T. Oja, Langmuir 8, 1211 (1992).

${ }^{17}$ R. J. Hunter, Zeta Potential in Colloid Science (Academic, London, 1981), Chap. 4.

${ }^{18}$ R. Pelton, P. Miller, W. McPhee, and S. Rajaram, Colloids Surf., A 80, 181 (1993).

${ }^{19}$ R. W. O'Brien and L. R. White, J. Chem. Soc., Faraday Trans. 2 74, 1607 (1978).

${ }^{20}$ J. C. Crocker and D. Grier, J. Colloid Interface Sci. 179, 298 (1996). 
${ }^{21}$ A.-P. Hynninen and M. Dijkstra, Phys. Rev. Lett. 94, 138303 (2005).

${ }^{22}$ W. W. Wood and J. D. Jacobson, J. Chem. Phys. 27, 1207 (1957); B. J. Alder and T. E. Wainwright, ibid. 27, 1208 (1957).

${ }^{23}$ B. Zoetekouw and R. van Roij, Phys. Rev. E 73, 021403 (2006).

${ }^{24}$ J. A. Weiss, A. E. Larsen, and D. G. Grier, J. Chem. Phys. 109, 8659 (1998).

${ }^{25}$ D. Derks, H. Wisman, A. van Blaaderen, and A. Imhof, J. Phys.: Condens. Matter 16, S3917 (2004); D. Derks, Ph.D. thesis, Utrecht University, 2006 (Available at http://www.colloid.nl).

${ }^{26}$ P. N. Pusey, W. van Megen, P. Bartlett, B. J. Ackerson, J. G. Rarity, and S. M. Underwood, Phys. Rev. Lett. 63, 2753 (1989); N. A. M. Verhaegh, J. S. van Duijneveldt, A. van Blaaderen, and H. N. W. Lekkerkerker, J. Chem. Phys. 102, 1416 (1995); M. D. Haw, W. C. K. Poon, and P. N.
Pusey, Phys. Rev. E 57, 6859 (1998); J. P. Hoogenboom, D. Derks, P. Vergeer, and A. van Blaaderen, J. Chem. Phys. 117, 11320 (2002).

${ }^{27}$ S. Auer and D. Frenkel, J. Phys.: Condens. Matter 14, 7667 (2002).

${ }^{28}$ E. J. Meijer and F. El Azhar, J. Chem. Phys. 106, 4678 (1997); F. El Azhar, M. Baus, J.-P. Ryckaert, and E. J. Meijer, ibid. 112, 5121 (2000).

${ }^{29}$ P.Pieranski, L. Strzelecki, and B. Pansu, Phys. Rev. Lett. 50, 900 (1983).

${ }^{30}$ S. Neser, C. Bechinger, P. Leiderer, and T. Palberg, Phys. Rev. Lett. 79, 2348 (1997); A. B. Fontecha, H. König, T. Palberg, R. Messina, and H. Löwen, J. Phys.: Condens. Matter 17, S2779 (2005).

${ }^{31}$ A. Fortini and M. Dijkstra, J. Phys.: Condens. Matter 18, L371 (2006). ${ }^{32}$ C. N. Likos, Phys. Rep. 348, 267 (2001).

${ }^{33}$ K. Zahn, A. Wille, G. Maret, S. Sengupta, and P. Nielaba, Phys. Rev. Lett. 90, 155506 (2003). 